\title{
Instrumentos de avaliação da atividade física em pacientes oncológicos: Uma revisão integrativa
}

\author{
Physical activity assessment instruments in cancer patients: An integrative review
}

\begin{abstract}
Instrumentos de evaluación de la actividad física en pacientes con cáncer: Una revisión integrativa
\end{abstract}

Elaine Cristine da Silva ${ }^{1 *}$, Magnus Benettii ${ }^{1}$, Joris Pazin ${ }^{1}$, Luani de Aguiar Silva ${ }^{1}$, Anderson Luis Faber Pereira ${ }^{1}$, Mariana da Silva de Aguiar ${ }^{1}$, Sabrina Fernandes de Azevedo', Paulo José Barbosa Gutierres Filho ${ }^{1}$, Milena Ketzer Caliendo dos Reis ${ }^{3}$, Thamara Caviquioni', Rudney da Silva ${ }^{1}$.

\section{RESUMO}

Objetivo: Identificar e analisar os principais instrumentos de avaliação da atividade física relacionados a diferentes condições associadas ao câncer. Métodos: A busca orientou-se pela estratégia SPIDER e utilizou descritores relativos à amostra: Sobreviventes de Câncer; aos fenômenos: Câncer/Saúde/Atividade Física/Exercício; ao delineamento: Teórico/Empírico/Metodológico; à avaliação do fenômeno: Pesquisas e questionários, e ao tipo de pesquisa: Qualitativa/Quantitativa. Foram utilizadas as bases MEDLINE, SciELO, LILACS e PEDro, além do Schoolar Google. A análise dos dados foi realizada através de ferramentas elementares de análise de conteúdo. Resultados: Os instrumentos de avaliação podem fornecer relevantes conhecimentos sobre diversas condições relacionadas à atividade física, principalmente, o nível de atividade física. Os monitores de atividade física apresentam maior validade e confiabilidade, mas maior custo financeiro, o que dificulta seu uso em estudos ou intervenções com grandes amostras. Os questionários apresentam menor precisão, podem ser aplicados em pacientes com diferentes tipos de câncer com adequada validade, e possuem baixo custo financeiro. Considerações finais: Existem diversos instrumentos de avaliação da atividade física, sendo que os questionários são mais utilizados porque, apesar de menor precisão, podem ser considerados uma opção válida e de baixo custo.

Palavras-chave: Saúde, Neoplasias, Exercício, Questionários.

\begin{abstract}
Objective: To identify and analyze the main physical activity assessment instruments related to different conditions associated with cancer. Methods: The search was guided by the SPIDER strategy and used descriptors related to the sample: Survivors of Cancer; to phenomena: Cancer / Health / Physical Activity / Exercise; to the design: Theoretical / Empirical / Methodological; to the evaluation of the phenomenon: Research and questionnaires, and to the type of research: Qualitative / Quantitative. The MEDLINE, SciELO, LILACS and PEDro databases were used, as well as the Google Schoolar. Data analysis was performed using elementary content analysis tools. Results: The evaluation instruments can provide relevant knowledge about various conditions related to physical activity, mainly the level of physical activity. The physical activity monitors present greater validity and reliability, but greater financial cost, which makes it difficult to use them in studies
\end{abstract}

${ }^{1}$ Universidade do Estado de Santa Catarina (UDESC), Florianópolis - SC.

*E-mail: elainecristine.cfd@gmail.com

2Universidade de Brasília (UNB), Brasília - DF.

${ }^{3}$ Centro Universitário de Maringá (UNICESUMAR), Maringá - PR.

SUBMETIDO EM: $3 / 2019$

ACEITO EM: $4 / 2019$

PUBLICADO EM: 7/2019

REAS/EJCH | Vol. 11(12) | e658 | DOI: https://doi.org/10.25248/reas.e658.2019 Página 1 de 8 
or interventions with large samples. The questionnaires are less accurate, can be applied in patients with different types of cancer with adequate validity, and have a low financial cost. Final considerations: There are several physical activity assessment instruments, and the questionnaires are more used because, despite less precision, they can be considered a valid and low-cost option.

Key words: Health, Neoplasms, Exercise, Questionnaires.

\section{RESUMEN}

Objetivo: Identificar y analizar los principales instrumentos de evaluación de la actividad física relacionados a diferentes condiciones asociadas al cáncer. Métodos: La búsqueda se orientó por la estrategia SPIDER y utilizó descriptores relativos a la muestra: Sobrevivientes de Cáncer; a los fenómenos: Cáncer / Salud / Actividad Física / Ejercicio; al delineamiento: Teórico / Empírico / Metodológico; a la evaluación del fenómeno: Investigaciones y cuestionarios, y al tipo de investigación: Cualitativa / Cuantitativa. Se utilizaron las bases MEDLINE, SciELO, LILACS y PEDRO, además del Schoolar Google. El análisis de los datos fue realizado a través de herramientas elementales de análisis de contenido. Resultados: Los instrumentos de evaluación pueden proporcionar relevantes conocimientos sobre diversas condiciones relacionadas a la actividad física, principalmente, el nivel de actividad física. Los monitores de actividad física presentan mayor validez y confiabilidad, pero mayor costo financiero, lo que dificulta su uso en estudios o intervenciones con grandes muestras. Los cuestionarios presentan menor precisión, pueden ser aplicados en pacientes con diferentes tipos de cáncer con adecuada validez, y tienen bajo costo financiero. Consideraciones finales: Existen diversos instrumentos de evaluación de la actividad física, siendo que los cuestionarios son más utilizados porque, a pesar de menor precisión, pueden ser considerados una opción válida y de bajo costo.

Palabras clave: Salud, Neoplasias, Ejercicio, Cuestionarios.

\section{INTRODUÇÃO}

O câncer é uma das principais causas de morte na atualidade. Neste contexto, pode-se apontar diversas terapêuticas medicamentosas e não medicamentosas que podem auxiliar tanto o tratamento quanto o acompanhamento de pacientes com diferentes tipos de câncer. Segundo estudos realizados, somente nos Estados Unidos, existem mais de 11 milhões de sobreviventes vivos. Evidências fornecidas na última década apontam que a realização de atividades físicas para sobreviventes do câncer, tanto durante quanto após o tratamento convencional, pode auxiliar sobre diversas condições associadas à esta doença, como por exemplo, a fadiga e a caquexia (SPECK RM et al., 2010).

Apresentando deste modo, a relevância do conhecimento adequado de questões ainda pouco abordadas na literatura da área da oncologia, como frequência, duração, intensidade, aplicações e limitações associadas, as quais devem ser devidamente consideradas tanto para a prática clínica quanto para a pesquisa científica (HUTZLER YS, 2006; HUTZLER YS, 2011; HENRIKSEN HB et al., 2018).

Segundo Camargo RZT (2015), estimativas do Instituto Nacional do Câncer dos Estados Unidos, no ano de 2014 mais de 1,6 milhões de pessoas foram diagnosticadas com câncer. Contudo, devido ao diagnóstico precoce e aos métodos de tratamento mais eficazes, é estimado que a incidência e a prevalência desta doença se elevem nos próximos anos.

No Brasil, estimativas do Instituto Nacional do Câncer José Gomes Alencar da Silva (INCA) indicavam que no período $2016 / 2017$ mais 500.000 novos casos de câncer seriam diagnosticados no país, sendo que $48,8 \%$ somente na região sudeste (INCA, 2017).

Os dados apontam ainda que do total das neoplasias, eram estimadas mais de 100.000 homens mortos e mais de 90.000 mulheres mortas em todo o Brasil (INCA, 2017). Algumas consequências podem ser identificadas neste contexto, como por exemplo, prevalências de doenças vasculares nos pacientes 
acometidos pelo câncer maiores que na população em geral. Esta maior propensão pode ser associada a fatores de risco como idade avançada e tabagismo, que se somam, por exemplo, aos efeitos colaterais do tratamento, como a cardiotoxicidade dos quimioterápicos e radioterapia (CAMARGO RZT, 2015).

Nos Estados Unidos 45\% dos sobreviventes de câncer têm mais de 70 anos e $64 \%$ tiveram o diagnóstico há mais de cinco anos (MERGEN CTT e STRASSBURGER SZ, 2009). No Brasil, 44.4\% das mortes ocorrem na faixa etária dos 70 anos ou mais (INCA, 2017).

Nos Estados Unidos, a neoplasia mais prevalente atualmente, excluindo-se o câncer de pele não melanoma, entre as mulheres sobreviventes é o câncer de mama (41\%) e entre os homens sobreviventes é o câncer de próstata (43\%) (MERGEN CTT e STRASSBURGER SZ, 2009).

No Brasil, a neoplasia mais prevalente entre as mulheres é o câncer de mama $(15,7 \%)$ e entre os homens é o câncer de traquéia, brônquios e pulmões (14,3\%) (INCA, 2017). Estas diferenças, ainda que nos Estados Unidos representem a prevalência entre sobreviventes e no Brasil entre óbitos relacionados ao câncer, pode refletir as condições de populações mais ou menos afluentes que acabam tendo correspondência com o observado na comparação de incidências desses tumores entre diferentes países, como os sistemas de saúde, ou as condições sociais, econômicas e educacionais, entre outras (FAGGIANO et al., 1997).

Neste preocupante cenário de saúde pública mundial e nacional, tem se destacado estratégias não farmacológicas de atendimento que podem colaborar nas diversas fases do tratamento associados ao câncer, como por exemplo, a prática de atividades físicas (HENRIKSEN HB et al., 2018).

A atividade física pode contribuir não somente na diminuição das taxas de mortalidade, mas no próprio atendimento aos efeitos do tratamento farmacológico tradicional. Esta estratégia vem sendo considerada um importante auxílio no tratamento não farmacológico, contribuindo diretamente e indiretamente no combate às deficiências fisiológicas e às condições psicológicas associadas, bem como nos efeitos do tratamento em pacientes com câncer, contribuindo deste modo para a qualidade de vida dos mesmos (SPECK RM et al., 2010; CAMARGO RZT, 2015; HENRIKSEN HB et al., 2018).

Estudos evidenciam ainda que pacientes com câncer que realizam exercício físico supervisionado apresentam uma melhora na qualidade de vida indiferentemente das características demográficas e clínicas durante e após o tratamento (BUFFART LM et al, 2017).

Assim, nas últimas décadas vem aumentando significativamente a quantidade de estudos demonstrando a necessidade da avaliação física válida e precisa aos pacientes oncológicos (CAMARGO RZT, 2015; HENRIKSEN HB et al., 2018).

Apesar da existência de diversos instrumentos de avaliação da atividade física em pacientes oncológicos, estudos apontam a relevância da validade e da precisão envolvidas, como por exemplo, dos acelerômetros triaxiais (HENRIKSEN HB et al., 2018).

Contudo, existem fatores econômicos e humanos que nem sempre permitem o uso viável deste tipo de instrumento. Henriksen HB et al., (2018) apontam que os acelerômetros triaxiais, apesar de fornecerem dados válidos e confiáveis, na maioria das vezes apresentam custos elevados que inviabilizam seu uso. Já os métodos de auto ou hétero relatos, como os questionários, são mais viáveis, principalmente pelo baixo custo financeiro, tempo reduzido e facilidade de aplicação.

Contudo, apesar de nos últimos anos diversos questionários têm sido desenvolvidos e validados para avaliar o nível de atividade física, nem todos eles são de fácil entendimento ou avaliam as especificidades da atividade física aplicada aos pacientes oncológicos. (HENRIKSEN HB et al., 2018)

Deste modo, considerando os impactos que a atividade física pode provocar em pacientes oncológicos, tanto na fase de tratamento quanto na fase de acompanhamento, em especial as vantagens e desvantagens dos principais instrumentos de avaliação da atividade física na atualidade, em especial, dos questionários, este estudo tem como objetivo, realizar uma revisão integrativa da literatura especializada sobre os principais instrumentos de avaliação da atividade física em pacientes de diferentes tipos de câncer.

REAS/EJCH | Vol. 11(12) | e658 | DOI: https://doi.org/10.25248/reas.e658.2019 Página 3 de 8 


\section{MÉTODOS}

\section{Diretrizes e recomendações}

Para tanto, foram adotadas as etapas propostas por Whittemore R e Knafl K (2005), neste caso, (1) identificação do problema; (2) busca na literatura; (3) avaliação das obras; (4) análise dos dados; e (5) apresentação dos dados.

\section{Identificação do problema}

Para identificação do problema foi utilizado como ponto de partida o artigo publicado no Journal of Cancer Survivals, volume 4, páginas 87 a 100, de 2010, intitulado "An update of controlled physical activity trials in câncer survivors: a systematic review and meta-analysis", de Rebecca M. Speck, Kerry S. Courneya, Louise C. Mâsse, Sue Duval e Kathryn H. Schmitz o qual aponta que o câncer atinge milhões de pessoas em todo o mundo e a atividade física é um importante tratamento adjuvante não farmacológico (SPECK RM et al., 2014).

\section{Busca na literatura}

A busca na literatura foi orientada a partir da definição inicial dos descritores primários que visaram a identificação de registros na língua portuguesa e inglesa, seguindo-se a estratégia SPIDER de Methley et al., (2014), quanto à amostra: Sobreviventes de Câncer (Cancer Survivors); aos fenômenos de interesse: Câncer (Cancer), Saúde (Health), Atividade Física (Physical Activity), Exercício (Exercise); ao delineamento: Estudo Teórico (Theoretical Study), Estudo Empírico (Empirical Study), Estudo Metodológico (Methodological Study); à avaliação do fenômeno: Pesquisas e questionários (Surveys and Questionnaires); e ao tipo de pesquisa: Método Qualitativo (Qualitative Method), Método Quantitativo (Quantitative Method).

A busca primária foi realizada em bases de dados científicas da área da saúde, neste caso, a MEDLINE (Medical Literature Analysis and Retrieval System on-line) via Pubmed, SciELO (Scientific Electronic Library Online), LILACS (Literatura Latino-Americana e do Caribe em Ciências da Saúde) e PEDro (Physiotherapy Evidence Database). Para a busca secundária, optou-se pelo motor de busca do Schoolar Google. Foram incluídas obras científicas que tratam dos descritores adotados; sem limite de data; disponíveis na língua portuguesa e na língua inglesa; dos tipos de artigos (empíricos, revisionais e metodológicos), editoriais, dissertações e teses. Foram excluídos artigos que abordavam temáticas que apesar de indiretamente estarem relacionadas à atividade física em pacientes oncológicos, ou não contemplavam especificamente a atividade física em seus diversos âmbitos.

\section{Avaliação das obras}

Considerando que a busca tinha potencial de grande variação de tipos de pesquisa, optou-se por classificação dos registros a partir de dois critérios básicos: (a) relevância para a temática alvo ( $0=$ sem relevância; $1=c o m$ moderada relevância; $2=c o m$ alta relevância): (b) rigor editorial e/ou metodológico ( $0=$ sem rigor; 1 =moderado rigor; $2=$ =alto rigor). Foram selecionadas as obras identificadas nas buscas eletrônicas que apresentavam pelo menos um ponto na avaliação da relevância em seus títulos. Após a seleção dos títulos, foi realizada uma avaliação preliminar de todas as obras selecionadas e aquelas que tiveram 2 pontos na avaliação da relevância e 1 ou 2 pontos na avaliação do rigor foram lidas integralmente e eventualmente utilizadas.

\section{Análise dos dados}

Para análise dos dados, após a seleção final das obras, optou-se por utilizar ferramentas elementares da análise de conteúdo (BARDIN, 2000). Para tanto, foram definidos sub-temas que buscaram compor o quadro operacional e que orientaram o processo de construção textual. Além disto, para os artigos que continham resultados relevantes a este estudo, quando selecionados para este estudo, optou-se por utilizar uma escala de sete pontos sobre o nível de evidências provenientes de: $1=$ revisões sistemáticas ou metanálises; 2=ECR's controlados; 3=EC's não randomizados; $4=e s t u d o s$ de coorte e de caso-controle; $5=$ revisões sistemáticas de estudos descritivos e qualitativos; $6=$ estudo de caso descritivo ou qualitativo; $7=$ opinião de autoridades e/ou relatórios de comitê de especialistas e de estudos acadêmicos, baseada em Zandonai AP et al., (2010). 


\section{Apresentação dos dados}

Para a apresentação dos resultados, optou-se por desenvolver uma análise textual que buscasse as interrelações dos dois blocos temáticos. No primeiro bloco buscou-se analisar brevemente os aspectos envolvidos na avaliação da atividade física em oncologia e no segundo os principais questionários de avaliação da atividade física e suas condições associadas. Assim, selecionou-se os cinco questionários mais referidos na literatura especializada sobre atividade física e algumas condições associadas, enfatizando-se sua finalidade, validade e utilização.

\section{RESULTADOS E DISCUSSÃO}

\section{Avaliação da atividade física em oncologia}

O câncer vem sendo descrito a partir de dois fatores, sendo eles genéticos (internos) ambientais (externos). Quanto aos fatores internos, em sua maioria, estão geneticamente determinados e possuem uma ligação direta com a capacidade que o organismo possui de se proteger de agressões externas e podem ainda interferir na capacidade de defesa do organismo. Estes fatores podem interagir de diversas maneiras e aumentar a probabilidade de transformar células normais em células malignas. Quanto aos fatores externos, podem estes estar relacionados às condições ambientais inerentes ao clima ou localização geográfica, mas também podem ter relação com as interações humanas típicas de hábitos de vida próprios do meio social e cultural na qual a pessoa encontra-se inserida, a exemplo da exposição solar excessiva, o tabagismo entre outros. (BRASIL, 2010; TESCHE L e FABRIZIO ATF, 2016).

Segundo Henriksen HB et al., (2018), vem acontecendo um aumento nas pesquisas destinadas a avaliar os efeitos da atividade física sobre a saúde de pacientes oncológicos, visando ainda examinar os efeitos benéficos da atividade física tanto durante o tratamento do câncer quanto no pós-tratamento. Camargo RZT (2015) afirma que o grupo mais estudado tem sido os afetados pelo câncer de mama, na qual seguem protocolos de intervenção em atividade aeróbica, de resistência, treino intervalado, supervisionados ou não, e em diferentes fases do tratamento. Contudo, destaca que apesar da relevância da atividade física como parte do arsenal terapêutico em pacientes com câncer, estudos sobre estas temáticas ainda são muito recentes, como por exemplo, com pacientes oncológicos que desenvolvem doenças cardiovasculares.

Sendo assim, no intuito de estimar a adesão às recomendações de atividade física, estudos noruegueses demonstram que é necessário se considerar a avaliação física por meio de ferramentas que sejam válidas e precisas. Henriksen HB et al., (2018) apontam que o uso de monitores que objetivam registrar a atividade física e apresentar dados resultantes do registro, vem aumentando significativamente nas últimas décadas, tendo em vista a validade e a confiabilidade desses dados, como a exemplo da intensidade do gasto energético. Contudo, a exemplo de estudos com grandes populações, a utilização de instrumentos de avaliação como os monitores de atividade, apresentam dois fatores relevantes sendo eles o custo elevado e tempo consumido pelo pesquisador (HENRIKSEN HB et al., 2018).

Neste sentido, deve-se destacar que tanto os custos quanto a viabilidade são importantes fatores a considerar na avaliação da atividade física. Assim, os métodos de auto-relato, principalmente aqueles realizados por meio de questionários tornam-se um dos mais viáveis, principalmente pelo baixo custo financeiro, tempo reduzido e facilidade de aplicação. Nos últimos anos diversos questionários têm sido desenvolvidos e validados para avaliar o nível de atividade física. Contudo, a aplicação de questionários longos em pacientes oncológicos não é adequada, pois estes são cansativos e acabam conflitando com os efeitos colaterais relacionados ao tratamento da doença, como fadiga e declínio funcional (HENRIKSEN HB et al., 2018).

Para tanto, se faz necessário o uso de questionários menores que contenham questões bem definidas, que sejam de fácil entendimento e que evidenciem as especificidades das atividades físicas voltadas aos pacientes oncológicos, podem ser mais viáveis para pesquisas com pacientes oncológicos, tendo em vista 0 desgaste resultante da doença. (HENRIKSEN HB et al., 2018). 
Sendo assim, métodos com custo mais baixo e de fácil aplicabilidade tornam-se importantes para avaliar a adesão às recomendações da atividade física. Portanto, o método do auto-relato, principalmente aquele realizado por meio de questionário, acaba por torna-se mais comum e atende às recomendações de baixo custo financeiro e otimização de tempo. Nos últimos 30 anos, diversos foram os questionários desenvolvidos e validados para avaliar o nível de atividade física. Importante mencionar que a aplicação de questionários longos em pacientes oncológicos torna-se cansativo e de difícil o entendimento, tendo em vista os efeitos colaterais relacionados ao tratamento da doença, bem como a fadiga e declínio funcional (HENRIKSEN HB et al., 2018).

Para tanto, destaca-se a necessidade do uso de questionários que contenham questões bem definidas, de fácil entendimento e que evidenciem as diferentes intensidades de atividades físicas para os pacientes oncológicos (HENRIKSEN HB et al., 2018).

Deste modo, considerando o grande número de instrumentos de avaliação da atividade física em pacientes oncológicos, o levantamento sistematizado na literatura especializada permitiu identificar os principais questionários utilizados nos últimos anos, neste caso, EORTC-QLC-C30; NORDIET-FFQ; HUNT-PAQ; POMS; e SCL-90-R.

\section{Principais questionários de avaliação da atividade física e condições associadas}

O levantamento sistematizado na literatura especializada permitiu identificar, entre outros, os seguintes questionários de avaliação da atividade física em pacientes oncológicos ou de diversas condições clínicas, psíquicas e físicas associadas a este constructo (FRANCESCHINI J et al., 2010; HENRIKSEN HB et al., 2018; CRUZ JFA e MOTA MP, 1997; DIMEO FC et al., 1999):

EORTC-QLC-C30: O instrumento EORTC-QLQ-C30, foi criado pela Organização Européia de Pesquisa e Tratamento do Câncer (EORTC) e tem três versões. Este instrumento é válido e muito utilizado para avaliar a qualidade de vida de pacientes com câncer e é composto por perguntas relacionadas a cinco escalas funcionais (física, funcional, emocional, social e cognitiva), uma escala sobre o estado de saúde global, três escalas de sintomas (fadiga, dor e náuseas/vômitos) e seis itens de sintomas adicionais (dispnéia, insônia, perda de apetite, constipação, diarréia e dificuldades financeiras).

Já a versão intitulada QLQ-LC13, acaba tendo uma especificidade, qual seja o uso para pacientes portadores de tumores de pulmão. Sendo assim, o QLQ-LC13 adiciona ao EORTC QLQ-C30, 13 questões relacionadas a uma escala de sintomas (dispnéia) e nove itens de sintomas e efeitos adversos ao tratamento como: tosse, hemoptise, mucosite, disfagia, neuropatia periférica, alopecia, dor no tórax, dor no braço ou ombro e dor em outras partes. Esse questionário foi validado no Brasil através de um estudo que teve como objetivo avaliar a qualidade de vida de pacientes com câncer colo-retal, tratados com intenção curativa (FRANCESCHINI J et al., 2010).

NORDIET-FFQ: O instrumento denominado NORDIET-FFQ teve o intuito de investigar o efeito de uma dieta semelhante à norueguesa, tratando ainda sobre a sobrevida global. O questionário de frequência semiquantitativa curta, encontra-se disponível mediante solicitação ao autor correspondente. $O$ instrumento teve como objetivo relatar a alimentação e o nível de atividade física nas últimas semanas, ou seja, nos últimos 1 ou 2 meses (HENRIKSEN HB et al., 2018).

A validade dos itens alimentares do NORDIET-FFQ foi publicada em diferentes países e como o protocolo para tratamento de dados, seguiram os mesmos procedimentos descritos em detalhes em Henriksen HB et. al, 2018.

Sendo assim, o NORDIET-FFQ teve alterações e foi completado pelos pacientes no centro de estudo. Os textos explicativos de ambas as perguntas sobre atividade física, incluíram exemplos de atividades típicas: intensidade moderada foi exemplificada por caminhada rápida, tarefas domésticas ou outras atividades resultando em leve falta de ar e intensidade vigorosa foi exemplificada por corrida, esqui cross-country ou outras atividades resultando em alta falta de ar (HENRIKSEN HB et al., 2018).

REAS/EJCH | Vol. 11(12) | e658 | DOI: https://doi.org/10.25248/reas.e658.2019 Página 6 de 8 
HUNT-PAQ: O questionário de atividade física norueguês intitulado HUNT-PAQ foi baseado no questionário usado no estudo HUNT 3 (Helseundersokelsen Nord Trondelag). Importante destacar que somente cinco questões sobre atividade física foram utilizadas. Para tanto os produtos de frequência e duração foram pesados pelo nível de intensidade, ou seja, Baixo, moderado ou Vigoroso codificado como 1, 2 e 3 , respectivamente.

O baixo nível de intensidade não foi avaliado, mas tem uma questão sobre o tempo sedentário diário em horas em um dia normal. A questão sobre frequência contém as seguintes respostas: Nunca e Menos que uma vez por semana, ambos codificados como 0 , uma vez por semana codificados como 1, 2-3 vezes por semana codificados como 2. 5, e Quase todos os dias codificados como 7.

A pergunta sobre a duração da atividade contém as respostas: Menos de 15 min codificados como 12 (subtraídos 20\% de 15), 15-29 min codificados como 22, 30-1 codificados como 45 e mais de $1 \mathrm{~h}$ codificados como 72 (adicionados 20\% para 60). Os produtos de frequência e duração são pesados pelo nível de intensidade, ou seja, Baixo, moderado ou Vigoroso codificado como 1, 2 e 3, respectivamente. Importante destacar que não houve validação do instrumento HUNT-PAQ para o contexto cultural e lingüístico do Brasil (HENRIKSEN HB et al., 2018).

POMS: O questionário intitulado Profile Of Moods States - POMS apresenta a versão curta e produz escores de subescala para depressão, fadiga, raiva/hostilidade e vigor, tendo como objetivo avaliar os efeitos psicológicos da atividade física (CRUZ JFA e MOTA MP, 1997).

O instrumento intitulado POMS, vem sendo utilizado em vários estudos clínicos para avaliar estados afetivos e sintomatologia mental em diferentes populações de pacientes e mostram alta confiabilidade e validade. Possui um total de 30 questões, na qual pretende estabelecer 0 índice de humor. Para tanto, 0 índice de longevidade e subescalas separadas para somatização, traços obsessivo-compulsivos, sensibilidade interpessoal, depressão, ansiedade, hostilidade, ansiedade fóbica, ideação paranóide e psicoticismo. Tendo uma versão Portugal, onde foi devidamente validado.

SCL-90-R: O questionário denominado SCL-90-R, busca avaliar o estado psicológico dos pacientes (DIMEO FC et al., 1999). Composto de afirmações sobre experiências subjetivas, o SCL-90-R permite a avaliação dos estados afetivos durante a última semana.

Assim, o SCL-90-R produz um índice de gravidade global e sub-escalas separadas para somatização, traços obsessivo-compulsivos, sensibilidade interpessoal, depressão, ansiedade, hostilidade, ansiedade fóbica, paranóide ideação e psicoticismo.

Escores mais altos indicam maior transtorno geral do humor; menores escores de vigor e maiores escores para as subescalas restantes indicam maior intensidade de distúrbio nas áreas examinadas. $O$ instrumento tem sido utilizado em vários estudos clínicos para avaliar estados afetivos e sintomatologia mental em diferentes populações de pacientes e têm mostrado alta confiabilidade e validade.

\section{CONSIDERAÇÕES FINAIS}

A revisão realizada na literatura especializada permitiu verificar que existem diversos tipos de instrumentos de avaliação de atividade física e suas condições associadas, e que a função e a utilização destes instrumentos dependem do tipo de estudo alvo, dos recursos envolvidos e das condições associadas. Também ficou evidente que os monitores de atividade física fornecem dados mais precisos e confiáveis, mas apresentam elevados custos, enquanto que os questionários são menos precisos, mas são mais fáceis de usar em pacientes oncológicos, principalmente pelas condições inerentes ao câncer ou seu tratamento convencional, como fadiga, enjôos, entre outros que podem estar presentes durante as coletas de dados. Além disto, foi possível constatar a existência diversos questionários de avaliação da atividade física e de diversas condições associadas ao tratamento e/ou atendimentos aos pacientes oncológicos, como o EORTC QLQ-C30 (qualidade de vida, aspectos funcionais, saúde global e sintomas), o NORDIET-FFQ (dietas), o HUNT-PAQ, o POMS (depressão, fadiga, raiva/hostilidade e vigor) e o SCL-90-R (estado psicológico).

REAS/EJCH | Vol. 11(12) | e658 | DOI: https://doi.org/10.25248/reas.e658.2019 Página 7 de 8 


\section{REFERÊNCIAS}

1. BARDIN L. Análise de conteúdo. Lisboa: Edições 70, 2000.

2. BRASIL. MINISTÉRIO DA SAÚDE. INSTITUTO NACIONAL DO CÂNCER - INCA. Como se comportam as células cancerosas. Rio de Janeiro: Instituto Nacional do Câncer, 2010.

3. BUFFART LM, et al. Effects and moderators of exercise on quality of life and physical function in patients with cancer: An individual patient data meta-analysis of 34 RCTs. Cancer treatment reviews, 2017; 52: 91-104.

4. CAMARGO RZT. Câncer: Mais um motivo para se exercitar. Revista DERC, 2015; 21(2): 58-60.

5. CRUZ JFA, MOTA MP. Adaptação e características psicométricas do "POMS - Profile of Moods States" e do "STAI - State Trait Anxiet Inventory". In: GONÇALVES M, et al. Avaliação psicológica: Formas e contexto. Volume 5, Braga: Associação dos Psicólogos Portugueses (APPORT), 1997.

6. DIMEO FC et al. Effects of physical activity on the fatigue and psychologic status of cancer patients during chemotherapy. Cancer, 1999; 85(10): 2273-2272.

7. FAGGIANO F, et al. Socioeconomic differences in cancer incidence and mortality. International Agency for Research on Cancer, 1997; 138: 65-176.

8. FRANCESCHINI J, et al. Reprodutibilidade da versão em português do Brasil do European Organization for Research and Treatment of Cancer Core Quality of Life Questionnaire em conjunto com seu módulo específico para câncer de pulmão. Jornal Brasileiro de Pneumologia, 2010; 36(5): 595-602.

9. HENRIKSEN HB, et al. Validation of two short questionnaires assessing physical activity in colorectal cancer patients. BMC Sports Science, Medicine and Rehabilitation, 2018; 10(2): 8.

10. INSTITUTO NACIONAL DE CÂNCER JOSÉ ALENCAR GOMES DA SILVA - INCA. ABC do câncer: abordagens básicas para o controle do câncer. org Mario Jorge Sobreira da Silva. 3. ed. rev. atual. Rio de Janeiro: Instituto Nacional de Câncer, 2017.

11. MERGEN CTT, STRASSBURGER SZ. Prevalência dos tipos de câncer em pacientes atendidos em um centro de alta complexidade em oncologia. Revista Contexto \& Saúde, 2009; 9(17): 31-38.

12. METHLEY AM, et al. PICO, PICOS and SPIDER: A comparison study of specificity and sensitivity in three search tools for qualitative systematic reviews. BMC Health Services Research, 2014; 14: 579.

13. SPECK RM, et al. An update of controlled physical activity trials in cancer survivors: A systematic review and meta-analysis. Journal of Cancer Survivorship: Research and Practice, 2010; 4(2): 87-100.

14. TESCHE L, FABRIZIO ATF. Atividade física pós-câncer: qualidade de vida - Um projeto social. Relato de experiência. In: XXI Jornada de Pesquisa - UNIJUÍ. 2016. Ijuí, Rio Grande do Sul.

15. WHITTEMORE R, KNAFL K. The integrative review: Updated methodology. Journal of Advanced Nursing, 2005; 52(5): 546-553.

16. ZANDONAI AP, et al. Qualidade de vida nos pacientes oncológicos: Revisão integrativa da literatura latinoamericana. Revista Eletrônica de Enfermagem, 2010; 12(3): 554-61. 